\title{
SHADOWING PROPERTY, WEAK MIXING AND REGULAR RECURRENCE
}

\author{
JIAN LI AND PIOTR OPROCHA
}

\begin{abstract}
We show that a non-wandering dynamical system with the shadowing property is either equicontinuous or has positive entropy and that in this context uniformly positive entropy is equivalent to weak mixing. We also show that weak mixing together with the shadowing property imply the specification property with a special kind of regularity in tracing (a weaker version of periodic specification property). This in turn implies that the set of ergodic measures supported on the closures of orbits of regularly recurrent points is dense in the space of all invariant measures (in particular, invariant measures in such a system form the Poulsen simplex, up to an affine homeomorphism).
\end{abstract}

\section{INTRODUCTION}

The notion of shadowing property (also known under the name of pseudo-orbit tracing property) was introduced in the fundamental works of Anosov and Bowen on hyperbolic aspect of differentiable dynamics. It was later discovered that this notion can lead to many interesting results in the study of measure theoretic and topological properties of dynamical systems on compact metric spaces. These studies are now among classical results of ergodic theory and qualitative theory of dynamical systems (see [3] and [7]).

In [16] Moothathu proved that in every non-wandering dynamical system with the shadowing property the set of minimal points is dense and recently this result was extended in [17, by showing that the set of regularly recurrent points is also dense. If a non-wandering dynamical system with the shadowing property is sensitive, various types of minimal subsystems are present in the dynamics, such as sensitive almost 1-1 extension of odometers and minimal subsystems with positive entropy. In fact, every non-empty open subset contains an extension of the full shift for some power of the action. It is shown in [16] that for a dynamical system with the shadowing property, a sensitive point from the non-wandering set is an entropy point and we will show that there is a full shift factor for some power of the action in every neighborhood of a sensitive point. As an immediate consequence we obtain a kind of dichotomy: a nonwandering dynamical system with the shadowing property is either equicontinuous or has positive entropy. We also obtain a few equivalent conditions for positive entropy in systems with the shadowing property. It is known (see [14]) that for a continuous map $f:[0,1] \rightarrow[0,1]$ weak mixing is equivalent to uniformly positive entropy of all orders.

Date: November 13, 2013.

2010 Mathematics Subject Classification. Primary: 37B05; Secondary: 37C50, 37B40.

Key words and phrases. shadowing property, pseudo-orbit, sensitivity, topological entropy, weak mixing, specification property. 
In the present paper we show that the same equivalence is true for non-trivial systems with the shadowing property.

It was first proved by Bowen that if a weakly mixing system has the shadowing property, then it satisfies the specification property and if additionally the system is positively expansive, then it satisfies the periodic specification property (e.g. see [7, Proposition 23.20]). We will combine this result with the above mentioned technique from [17] to show that if a dynamical system with the shadowing property is weakly mixing then it has the specification property with some regularity in tracing.

It is shown in [20] that if a dynamical system satisfies the periodic specification property, then the set of atomic measures which are uniformly distributed on the periodic orbits is dense in the set of invariant measures. This classical result was later extended by various authors in many different settings, including maps on the unit interval [9] where there is no chance for any kind of expansiveness. It is also known that positively expansive systems with the shadowing property have dense sets of periodic points. But there are also weakly mixing systems with the shadowing property and without periodic points (e.g. see Example [5.6), therefore they are not positively expansive. While there is no possibility to obtain the same result (e.g. on the structure of the sets of invariant measures) in general setting, some part of qualitative behavior survives if we drop the positive expansiveness assumption. To be specific, we show that if a weakly mixing system has the shadowing property, then the set of ergodic invariant measures supported on the closures of orbits of regularly recurrent points is dense in the set of invariant measures. Since extreme points of invariant measure are exactly the ergodic measures, invariant measures in such a system form the Poulsen simplex, up to an affine homeomorphism. For more details about the Poulsen simplex and its connections with dynamical systems, we refer the reader to [11] and [15].

\section{Preliminaries}

Throughout this paper, let $\mathbb{N}, \mathbb{N}_{0}$ and $\mathbb{R}$ denote the set of all positive integers, nonnegative integers and real numbers, respectively. Let $(X, d)$ be a metric space. Open and closed balls of radius $r>0$ centered at a point $x$ in $X$ are denoted by $B(x, r)$ and $\bar{B}(x, r)$, respectively. A subset $A$ of $X$ is residual if it is a dense $G_{\delta}$ set.

2.1. Topological dynamics. A dynamical system is a pair $(X, f)$, where $X$ is a compact metric space with a metric $d$ and $f: X \rightarrow X$ is a continuous map.

The orbit of a point $x \in X$ is the set $\operatorname{Orb}(x, f)=\left\{f^{n}(x): n \in \mathbb{N}_{0}\right\}$. The set of limit points of the orbit $\operatorname{Orb}(x, f)$ is called the $\omega$-limit set of $x$, and is denoted by $\omega(x, f)$.

A subset $D$ of $X$ is $f$-invariant (or simply invariant) if $f(D) \subset D$. A non-empty closed invariant subset $D$ of $X$ is minimal, if $\overline{\operatorname{Orb}(x, f)}=D$ for every $x \in D$. A point $x \in X$ is minimal if it is contained in some minimal subset of $X$.

A point $x \in X$ is periodic with least period $n$ if $n$ is the smallest positive integer satisfying $f^{n}(x)=x$; recurrent if for every neighborhood $U$ of $x$ there exists $k \in \mathbb{N}$ such that $f^{k}(x) \in U$; regularly recurrent if for every open neighborhood $U$ of $x$, there exists $k \in \mathbb{N}$ such that $f^{k n}(x) \in U$ for all $n \in \mathbb{N}_{0}$. It is well known that a point $x \in X$ is minimal if and only if for every open neighborhood $U$ of $x$, there exists $N>0$ such that $f^{k}(x) \in U$ for some $k \in[n, n+N]$ and every $n \in \mathbb{N}_{0}$. Note that every periodic point is 
regularly recurrent, every regularly recurrent point is minimal and every minimal point is recurrent (but not vice-versa). Denote by $P(f), R(f), M(f)$ and $R R(f)$, respectively, the set of all periodic, recurrent, minimal and regularly recurrent points of $f$. We say that $x \in X$ is a non-wandering point if for every neighborhood $U$ of $x$, there exists $k \in \mathbb{N}$ such that $f^{k}(U) \cap U \neq \emptyset$. The set of all non-wandering points of $f$ is denoted as $\Omega(f)$. Observe that $\Omega(f)$ is closed and $f$-invariant. If $\Omega(f)=X$, the system is said to be non-wandering.

We say that $f$ is transitive if for every pair of non-empty open subsets $U$ and $V$ of $X$ there is $k \in \mathbb{N}$ such that $f^{k}(U) \cap V \neq \emptyset$; weakly mixing if $f \times f$ is transitive; strongly mixing if for every pair of non-empty open sets $U$ and $V$ of $X$ there is an $N>0$ such that $f^{n}(U) \cap V \neq \emptyset$ for all $n \geq N$. We say that $x \in X$ is a transitive point if $\omega(x, f)=X$. It is well known that a dynamical system $(X, f)$ is transitive if and only if the set of transitive points is residual in $X$.

A dynamical system $(X, f)$ is equicontinuous if for every $\varepsilon>0$, there is $\delta>0$ with the property that $d(x, y)<\delta$ implies $d\left(f^{n}(x),, f^{n}(y)\right)<\varepsilon$ for every $n \in \mathbb{N}$ and every $x, y \in X$. A point $x \in X$ is equicontinuous if for every $\varepsilon>0$, there is $\delta>0$ with the property that $d(x, y)<\delta$ implies $d\left(f^{n}(x),, f^{n}(y)\right)<\varepsilon$ for every $n \in \mathbb{N}$ and every $y \in X$. By the compactness of $X$, a system $(X, f)$ is equicontinuous if and only if every point in $X$ is equicontinuous.

A dynamical system $(X, f)$ is sensitive if there exists $\delta>0$ such that for any nonempty open subset $U$ of $X$, we have $\operatorname{diam}\left(f^{n}(U)\right)>\delta$ for some $n \in \mathbb{N}$. A point $x \in X$ is sensitive if it is not equicontinuous, that is there exists $\delta>0$ such that for any neighborhood $U$ of $x$ there is $n>0$ such that $\operatorname{diam}\left(f^{n}(U)\right)>\delta$. Clearly, if $(X, f)$ is sensitive then every point $x \in X$ is sensitive.

Let $(X, f)$ and $(Y, g)$ be two dynamical systems. If there is a continuous surjection $\pi: X \rightarrow Y$ with $\pi \circ f=g \circ \pi$, then we say that $\pi$ is a factor map, the system $(Y, g)$ is a factor of $(X, f)$ or $(X, f)$ is an extension of $(Y, g)$. We say that $(X, f)$ is almost 1-1 extension of $(Y, g)$ if $Z=\left\{x \in X: \pi^{-1}(\pi(x))=\{x\}\right\}$ is residual in $X$. If $\pi$ is a homeomorphism, then we say that $\pi$ is a conjugacy and dynamical systems $(X, f)$ and $(Y, g)$ are conjugate. Conjugate dynamical systems can be considered the same from the dynamical point of view.

2.2. Shifts and Odometers. For any integer $d \geq 1$, the space $\{0,1, \ldots, d\}^{\mathbb{N}_{0}}$ is a Cantor space with respect to the product topology. We write elements of $\{0,1, \ldots, d\}\}^{\mathbb{N}_{0}}$ as $\alpha=a_{0} a_{1} a_{2} \ldots$ The shift map $\sigma:\{0,1, \ldots, d\}^{\mathbb{N}_{0}} \rightarrow\{0,1, \ldots, d\}^{\mathbb{N}_{0}}$ is defined by the condition that $\sigma(\alpha)_{n}=\alpha_{n+1}$ for $n \in \mathbb{N}_{0}$. It is not hard to check that $\sigma$ is a continuous surjection. The dynamical system $\left(\{0,1, \ldots, d\}^{\mathbb{N}_{0}}, \sigma\right)$ is called the full shift.

Let $s=\left(s_{j}\right)_{j=1}^{\infty}$ be a sequence of positive integers such that $s_{j}$ divides $s_{j+1}$. Let $X(j)=\left\{0,1, \ldots, s_{j}-1\right\}$ and $X_{s}=\left\{x \in \prod_{j=1}^{\infty} X(j): x_{j+1} \equiv x_{j}\left(\bmod s_{j}\right)\right\}$. Let $f: X_{s} \rightarrow X_{s}, x \mapsto y$, where $y_{j}=x_{j}+1\left(\bmod s_{j}\right)$ for each $j=1,2, \ldots$ The dynamical system $\left(X_{s}, f\right)$ is called an odometer (or an adding machine) defined by the sequence $s=\left(s_{j}\right)_{j=1}^{\infty}$. Some authors require that the sequence $\left(s_{j}\right)_{j=1}^{\infty}$ starts with at least 2 and is strictly increasing, but lack of such restrictions has no formal consequences other than admitting (as odometers) periodic orbits including the trivial one. It is clear that $\left(X_{s}, f\right)$ is minimal and equicontinuous, and every point in $X_{s}$ is regularly recurrent. 
In the further parts of the paper we will refer to the fact (e.g. see [8, Theorem 5.1]) that the orbit closure $\overline{\operatorname{Orb}(x, f)}$ of any regularly recurrent point $x \in X$ is an almost 1-1 extension of an odometer. See the survey [8] by Downarowicz for more details on odometers.

2.3. The shadowing property. Let $(X, f)$ be a dynamical system. Fix any $\varepsilon>0$ and $\delta>0$. A sequence $\left\{x_{n}\right\}_{n=0}^{\infty}$ in $X$ is a $\delta$-pseudo orbit if $d\left(f\left(x_{n}\right), x_{n+1}\right)<\delta$ for all $n=0,1,2, \ldots$ A point $x \in X$ is $\varepsilon$-tracing a pseudo-orbit $\left\{x_{n}\right\}_{n=0}^{\infty}$ if $d\left(f^{n}(x), x_{n}\right)<\varepsilon$ for all $n=0,1, \ldots$ We say that the system $(X, f)$ (or the map $f$ ) has the shadowing property (or pseudo-orbit tracing property) if for every $\varepsilon>0$ there is $\delta>0$ such that every $\delta$-pseudo orbit of $f$ is $\varepsilon$-traced by some point in $X$.

If $x, y \in X$ then an $\varepsilon$-chain of length $n>1$ (a finite $\varepsilon$-pseudo orbit) form $x$ to $y$ is any sequence $x_{1}, \ldots, x_{n}$ such that $x_{1}=x, x_{n}=y$ and $d\left(f\left(x_{i}\right), x_{i+1}\right)<\varepsilon$ for $i=1,2, \ldots, n-1$. We say that $(X, f)$ is chain mixing if for every $\varepsilon>0$ there is $M>0$ such that for any two points $x, y \in X$ and any $n \geq M$ there is an $\varepsilon$-chain of length $n$ from $x$ to $y$.

The following facts highlight strong connections between the non-wandering set and the shadowing property.

Theorem 2.1 ([3, 16]). If $f$ has the shadowing property, then $\left.f\right|_{\Omega(f)}$ also has the shadowing property.

Additionally, in dynamical systems with the shadowing property there are many minimal points.

Theorem 2.2 ([16]). Let $(X, f)$ be a non-wandering dynamical system with the shadowing property. Then the set $M(f)$ of minimal points is dense in $X$.

Theorem $2.3([17])$. Let $(X, f)$ be a non-wandering dynamical system with the shadowing property. Then the set $R R(f)$ of regularly recurrent points is dense in $X$.

The following fact can be easily deduced from known results. We present a proof here for completeness.

Proposition 2.4. Let $(X, f)$ be a non-wandering system. If $(X, f)$ is equicontinuous then $M(f)=X$ (i.e. $X$ is a union of minimal equicontinuous systems). If additionally $(X, f)$ has the shadowing property then $R R(f)=X$ (and each minimal subsystem is conjugated to an odometer).

Proof. Fix a point $x \in X$. Then it is not hard to see that $x$ is a recurrent point (see [2]). This implies (e.g. again by [2]) that $(\overline{O r b(x, f)}, f)$ is an equicontinuous minimal subsystem of $(X, f)$. By [16] any equicontinuous surjective dynamical $(X, f)$ has the shadowing property if and only if $X$ is totally disconnected. Then $(\overline{\operatorname{Orb}(x, f)}, f)$ is either a finite set (a periodic orbit) or a Cantor set, and then it is conjugate to an odometer (see [1] or [18]). In each case $x$ is regularly recurrent. 


\section{Dichotomy RESUlts FOR DYNAMiCAL SYSTEMS WiTH THE SHADOWING PROPERTY}

It is shown in [16] that for a dynamical system with the shadowing property, a sensitive point from the non-wandering set is an entropy point (see [22] for the definition). In fact, we can show that there is a full shift factor in every neighborhood of a sensitive point.

Proposition 3.1. Let $(X, f)$ be a dynamical system with the shadowing property. If $u \in X$ is a sensitive point of $(\Omega(f), f)$, then for every neighborhood $U$ of $u$, there exists a positive integer $m$, a subsystem $\left(Y, f^{m}\right)$ of $\left(X, f^{m}\right)$ with $Y \subset U$ and a factor map $\pi:\left(Y, f^{m}\right) \rightarrow\left(\{0,1\}^{\mathbb{N}_{0}}, \sigma\right)$.

Proof. Since $u$ is a sensitive point, there is a positive number $\lambda$ with the property that for any neighborhood $V$ of $u$, we have $\operatorname{diam}\left(f^{n}(V \cap \Omega(f))\right)>\lambda$ for some $n \in \mathbb{N}$. Pick a positive number $\varepsilon<\lambda / 8$ such that $B(u, 3 \varepsilon) \subset U$. By Theorem $2.1(\Omega(f), f)$ also has the shadowing property. Let $\delta$ be a constant provided for $\varepsilon>0$ by the shadowing property of $\left.f\right|_{\Omega(f)}$. By the definition of $\lambda$, there exists a positive integer $r$ and two points $v_{1}, v_{2} \in \Omega(f) \cap B(u, \delta / 4)$ such that $d\left(f^{r}\left(v_{1}\right), f^{r}\left(v_{2}\right)\right)>\lambda$. By Theorem $2.2(\Omega(f), f)$ has a dense set of minimal points, the same is true for $(\Omega(f) \times \Omega(f), f \times f)$. By the continuity of $f^{r}$, there exists a minimal point $\left(w_{1}, w_{2}\right)$ of $(\Omega(f) \times \Omega(f), f \times f)$ such that $d\left(v_{1}, w_{1}\right)<\delta / 4, d\left(v_{2}, w_{2}\right)<\delta / 4$ and $d\left(f^{r}\left(v_{1}\right), f^{r}\left(w_{1}\right)\right)<\varepsilon, d\left(f^{r}\left(v_{2}\right), f^{r}\left(w_{2}\right)\right)<\epsilon$. Then $d\left(w_{1}, w_{2}\right)<\delta$ and $d\left(f^{r}\left(w_{1}\right), f^{r}\left(w_{2}\right)\right)>\lambda-2 \epsilon>4 \varepsilon$. By the recurrence of $\left(w_{1}, w_{2}\right)$ there exists a positive integer $m>r$ such that $d\left(w_{1}, f^{m}\left(w_{1}\right)\right)<\delta / 4$ and $d\left(w_{2}, f^{m}\left(w_{2}\right)\right)<\delta / 4$. Define two finite sequences as follows

$$
\begin{aligned}
& \eta(0)=\left(w_{1}, f\left(w_{1}\right), \ldots, f^{m-1}\left(w_{1}\right)\right), \\
& \eta(1)=\left(w_{2}, f\left(w_{2}\right), \ldots, f^{m-1}\left(w_{2}\right)\right) .
\end{aligned}
$$

Let $W_{0}=\bar{B}\left(f^{r}\left(w_{1}\right), \varepsilon\right)$ and $W_{1}=\bar{B}\left(f^{r}\left(w_{2}\right), \varepsilon\right)$. Then $W_{0}$ and $W_{1}$ are non-empty closed subsets, and $\operatorname{dist}\left(W_{0}, W_{1}\right)>\epsilon$.

For every $\alpha=a_{0} a_{1} \ldots a_{n} \ldots \in\{0,1\}^{\mathbb{N}_{0}}$, we set

$$
Y_{\alpha}=\left\{x \in \bar{B}(u, 2 \varepsilon): f^{m i}(x) \in \bar{B}(u, 2 \varepsilon) \text { and } f^{m i+r}(x) \in W_{a_{i}} \text { for } i=0,1, \ldots\right\} .
$$

It is clear that every $Y_{\alpha}$ is a closed subset of $X$, and $Y_{\alpha} \cap Y_{\beta}=\emptyset$ for $\alpha \neq \beta$. We first show that every $Y_{\alpha}$ is not empty. Let $y_{\alpha}$ be a point $\varepsilon$-tracing the $\delta$-pseudo orbit $\eta\left(a_{0}\right) \eta\left(a_{1}\right) \cdots \eta\left(a_{n}\right) \cdots$. Then it is easy to verify that $y_{\alpha} \in Y_{\alpha}$.

Let $Y=\bigcup_{\alpha \in\{0,1\}^{\mathbb{N}_{0}}} Y_{\alpha}$. Then $Y \subset U$. We show that $\left(Y, f^{m}\right)$ is a subsystem of $\left(X, f^{m}\right)$. Let $\left\{y_{n}\right\}$ be a sequence in $Y$ and $y_{n} \rightarrow y$ as $n \rightarrow \infty$. For every $y_{n}$, there exists $\alpha_{n}$ such that $y_{n} \in Y_{\alpha_{n}}$. By the compactness of $\{0,1\}^{\mathbb{N}_{0}}$, without loss of generality we assume that $\alpha_{n} \rightarrow \alpha \in\{0,1\}^{\mathbb{N}_{0}}$. Then $y \in Y_{\alpha}$, which implies that $Y$ is closed. Now we show that $Y$ is $f^{m}$-invariant. Let $y \in Y$. Then there exists an $\alpha=a_{0} a_{1} \ldots a_{n} \ldots \in\{0,1\}^{\mathbb{N}_{0}}$ such that $y \in Y_{\alpha}$. By the definition of $Y_{\alpha}, f^{m i}(y) \in \bar{B}(u, 2 \varepsilon)$ and $f^{m i+r}(y) \in W_{a_{i}}$ for $i=0,1, \ldots$ Then $f^{m i}\left(f^{m}(y)\right) \in \bar{B}(u, 2 \varepsilon)$ and $f^{m i+r}\left(f^{m}(y)\right) \in W_{a_{i}+1}$ for $i=0,1, \ldots$, that is $f^{m}(y) \in Y_{\sigma(\alpha)} \subset Y$.

Now define a map $\pi: Y \rightarrow\{0,1\}^{\mathbb{N}_{0}}$ by $\pi\left(Y_{\alpha}\right)=\{\alpha\}$ for every $\alpha \in\{0,1\}^{\mathbb{N}_{0}}$. Then $\pi$ is a factor map between $\left(Y, f^{m}\right)$ and $\left(\{0,1\}^{\mathbb{N}_{0}}, \sigma\right)$. 
Remark 3.2. Consider the map $f:[0,1] \rightarrow[0,1], x \mapsto x^{2}$. It is well known that if an interval map has fixed points only at endpoints then it has the shadowing property (e.g. see [12, Lemma 4.1]). Then $([0,1], f)$ has the shadowing property. It is clear that 1 is a sensitive point, but $\Omega(f)=\{0,1\}$, and therefore we cannot apply Proposition 3.1 to $f$ in this case.

Let $h(X, f)$ denote the topological entropy of $(X, f)$. We refer the reader to the textbooks [7] or [21] for basic properties of topological entropy. By Proposition 3.1, we have the following dichotomy on non-wandering systems with the shadowing property. Note that this result is also essentially contained in [16, Corollary 5(i)].

Theorem 3.3. Let $(X, f)$ be a non-wandering dynamical system with the shadowing property. Then either $(X, f)$ is equicontinuous or $(X, f)$ has positive entropy.

Proof. If $(X, f)$ is not equicontinuous, then there exists a sensitive point in $X$. Since $(X, f)$ is non-wandering, $\Omega(f)=X$. Then by Proposition 3.1, there exists a positive integer $m$, a subsystem $\left(Y, f^{m}\right)$ of $\left(X, f^{m}\right)$ and a factor map $\pi:\left(Y, f^{m}\right) \rightarrow\left(\{0,1\}^{\mathbb{N}_{0}}, \sigma\right)$. Therefore, $h(X, f)=\frac{1}{m} h\left(X, f^{m}\right) \geq \frac{1}{m} h\left(\{0,1\}^{\mathbb{N}_{0}}, \sigma\right)>0$.

Lemma 3.4. Let $(X, f)$ be a dynamical system. If there exists a positive integer $m$ and a subsystem $\left(Y, f^{m}\right)$ of $\left(X, f^{m}\right)$ such that $\left(Y, f^{m}\right)$ is an extension of $\left(\{0,1\}^{\mathbb{N}_{0}}, \sigma\right)$, then $(\Omega(f) \backslash R(f)) \cap Y \neq \emptyset,(R(f) \backslash M(f)) \cap Y \neq \emptyset$ and $(M(f) \backslash R R(f)) \cap Y \neq \emptyset$.

Proof. Let $\pi:\left(Y, f^{m}\right) \rightarrow\left(\{0,1\}^{\mathbb{N}_{0}}, \sigma\right)$ be the factor map. Using Kuratowski-Zorn Lemma there is a closed and $f^{m}$-invariant set $\Lambda \subset Y$ such that $\pi(\Lambda)=\{0,1\}^{\mathbb{N}_{0}}$ and if $Z \subset \Lambda$ is a closed and $f^{m}$-invariant subset such that $\pi(Z)=\{0,1\}^{\mathbb{N}_{0}}$ then $Z=\Lambda$. Let $q \in\{0,1\}^{\mathbb{N}_{0}}$ be a point with dense orbit under $\sigma$ and fix $y \in \pi^{-1}(q) \cap \Lambda$. There is an increasing sequence $\left\{n_{i}\right\}$ such that $\lim _{i \rightarrow \infty} \sigma^{n_{i}}(q)=q$ and we can also assume, passing to a subsequence if necessary, that the $\operatorname{limit}_{\lim _{i \rightarrow \infty}} f^{m n_{i}}(y)=z$ exists. But then if we put $Z=\operatorname{Orb}\left(z, f^{m}\right) \subset \omega\left(y, f^{m}\right)$ then $q \in \pi(Z)$ and so $\pi(Z)=\{0,1\}^{\mathbb{N}_{0}}$. It immediately implies that $y \in \omega\left(y, f^{m}\right)=\Lambda$, and hence $\left(\Lambda, f^{m}\right)$ is transitive. Since the orbit of $y$ is dense in $\left(\Lambda, f^{m}\right)$ and $\left(\{0,1\}^{\mathbb{N}_{0}}, \sigma\right)$ is not minimal, we see that $y \in R(f) \backslash M(f)$. If we take, $\alpha=1000 \ldots \in \Omega(\sigma) \backslash R(\sigma)$, then $\pi^{-1}(\alpha) \subset \Omega\left(\Lambda, f^{m}\right) \backslash R\left(f^{m}\right) \subset \Omega(f) \backslash R(f)$, since $R(f)=R\left(f^{m}\right)$. Finally, if $(Z, \sigma)$ is a weakly mixing minimal system in $\left(\{0,1\}^{\mathbb{N}_{0}}, \sigma\right)$ (e.g. a Chacón flow) and $z$ is any minimal point in $\pi^{-1}(Z)$ then $z \in M\left(f^{m}\right) \backslash R R\left(f^{m}\right)=$ $M(f) \backslash R R(f)$.

It is shown in [17] that the set of regularly recurrent points is dense if $(X, f)$ is a non-wandering system with the shadowing property. If the system $(X, f)$ is also sensitive, then $M(f) \backslash R R(f)$ is dense in $X$. We can improve the conclusion as follows (Example 3.6 below shows that while every sensitive system has a dense set of sensitive points, the converse is not true).

Proposition 3.5. Let $(X, f)$ be a non-wandering system with the shadowing property. If $(X, f)$ has a dense set of sensitive points, then sets $\Omega(f) \backslash R(f), R(f) \backslash M(f)$ and $M(f) \backslash R R(f)$ are dense in $X$.

Proof. Fix a non-empty open subset $U$ of $X$. There exists a sensitive point $u \in U$. By Proposition [3.1, there exists a positive integer $m$, a subsystem $\left(Y, f^{m}\right)$ of $\left(X, f^{m}\right)$ 
with $Y \subset U$ and a factor map $\pi:\left(Y, f^{m}\right) \rightarrow\left(\{0,1\}^{\mathbb{N}_{0}}, \sigma\right)$. Then the result follows by Lemma 3.4.

Example 3.6. Let $T:[0,1] \rightarrow[0,1]$ be the standard tent map, that is $T(x)=1-|1-2 x|$. Let

$$
X=\{(0,0)\} \cup \bigcup_{k=1}^{\infty}\left\{\frac{1}{k}\right\} \times\left[0, \frac{1}{k}\right]
$$

be endowed with metric induced by the Euclidean metric and put

$$
f\left(\frac{1}{k}, x\right)=\left\{\begin{array}{ll}
\left(\frac{1}{k}, \frac{1}{k} T(k x)\right), & \text { for } k>0 \\
(0,0), & \text { otherwise }
\end{array} .\right.
$$

Then this map has the shadowing property. Simply, if we fix $\varepsilon>0$ then there is $\delta>0$ such that if $\left(\frac{1}{k}, x\right),\left(\frac{1}{s}, y\right) \in X$ and $\frac{1}{k}>\sqrt{\varepsilon}$ then either $k=s$ or $\left|\frac{1}{k}-\frac{1}{s}\right|>\delta$. This immediately implies that for any $\delta$-pseudo-orbit $\xi=\left\{z_{n}\right\}_{n=0}^{\infty}=\left\{\left(\frac{1}{k_{n}}, x_{n}\right)\right\}_{n=0}^{\infty}$ we either have that $\xi \subset[0, \sqrt{\varepsilon}] \times[0, \sqrt{\varepsilon}]$ or $k_{n}=k_{n+1}$ for every $n \geq 0$. But in the first case $(0,0)$ is an $\varepsilon$-tracing point for $\xi$ and in the second case we can use the shadowing property of $T$ (after appropriate rescaling of the sequence $\left.\left\{x_{n}\right\}_{n=0}^{\infty}\right)$. This proves that $f$ has the shadowing property but it is also clear that $f$ is equicontinuous at $(0,0)$.

Now we can provide a series of conditions equivalent, for systems with the shadowing property, to positive entropy.

Theorem 3.7. Let $(X, f)$ be a dynamical system with the shadowing property. Then the following conditions are equivalent:

(1) $(X, f)$ has positive entropy;

(2) there exists a sensitive transitive subsystem $(M, f)$ of $(X, f)$;

(3) there exists a sensitive point in $(\Omega(f), f)$;

(4) there exists a positive integer $m$, a subsystem $\left(Y, f^{m}\right)$ for $f^{m}$ and a factor map $\pi:\left(Y, f^{m}\right) \rightarrow\left(\{0,1\}^{\mathbb{N}_{0}}, \sigma\right) ;$

(5) $\Omega(f) \backslash R(f)$ is not empty;

(6) $R(f) \backslash M(f)$ is not empty;

(7) $M(f) \backslash R R(f)$ is not empty.

Proof. (11) $\Rightarrow$ (2) By the variation principle of topological entropy, there exists an ergodic invariant Borel probability measure $\mu$ on $X$ such that the measure-theoretic entropy of $\mu$ is positive. Denote by $M$ the support of $\mu$. By [10], $(M, f)$ is either sensitive or equicontinuous (it is so called E-system, i.e. transitive map with fully supported measure). Then the only possibility is that $(M, f)$ is sensitive, since it has positive entropy.

(2) $\Rightarrow$ (3) Let $x \in M$. Then $x$ is a sensitive point of $(M, f)$. Since $M \subset \Omega(f), x$ is also a sensitive point of $(\Omega(f), f)$.

(3) $\Rightarrow$ (4) follows from Proposition 3.1.

(44) $\Rightarrow$ (11) follows by $h(X, f)=\frac{1}{m} h\left(X, f^{m}\right) \geq \frac{1}{m} h\left(\{0,1\}^{\mathbb{N}_{0}}, \sigma\right)>0$.

(44) $\Rightarrow$ (5, 67) Applying Lemma 3.4, we obtain that $\Omega(f) \backslash R(f), R(f) \backslash M(f)$, and $M(f) \backslash R R(f)$ are non-empty. 
Finally, observe that if $(X, f)$ has zero topological entropy, then $(\Omega(f), f)$ also has zero entropy by the variational principle. By Theorem 3.3. $(\Omega(f), f)$ must be equicontinuous since $(\Omega(f), f)$ also has the shadowing property by Theorem 2.1 . In particular, in this situation we have $\Omega(f)=R(f)=M(f)=R R(f)$ by Proposition [2.4, which proves (5) $\Rightarrow(\mathbb{1}),([6) \Rightarrow$ (1) and (7) $\Rightarrow$ (1) completing the proof.

\section{WEAKLY MIXING SYSTEMS WITH THE SHADOWING PROPERTY}

Recall that a dynamical $(X, f)$ is called weakly mixing if $(X \times X, f \times f)$ is transitive. By the well known Furstenberg Intersection Lemma, if $(X, f)$ is weakly mixing, then $\left(X^{n}, f^{(n)}\right)$ is transitive for all $n \in \mathbb{N}$, where $X^{n}=X \times X \times \cdots \times X$ (n-times) and $f^{(n)}=f \times f \times \cdots \times f(n$-times $)$.

It is shown in [14] that for a continuous map $f:[0,1] \rightarrow[0,1]$ weak mixing is equivalent to uniformly positive entropy of all orders. The main result of this section is that the similar result also holds for non-trivial systems with the shadowing property.

Definition 4.1 ([4, 13]). A dynamical system $(X, f)$ is said to have uniformly positive entropy of all orders if for every $n \geq 2$, any cover of $X$ by $n$ non-dense open sets has positive entropy.

A system with uniformly positive entropy of all orders is a topological analogue of the Kolmogorov system in ergodic theory, so such a system is also called a topological $K$ system.

Definition $4.2([4,13])$. A dynamical system $(X, f)$ is said to have the strong Property $P$ if for any $n \geq 2$ and any non-empty open subsets $U_{0}, U_{1}, \ldots, U_{n-1}$ of $X$ there exists an integer $N$ such that whatever $k \geq 2$, whatever $s=(s(1), s(2), \ldots, s(k)) \in\{0,1, \ldots, n-$ $1\}^{k}$, there exists $x \in X$ with $x \in U_{s(1)}, f^{N}(x) \in U_{s(2)} \ldots, f^{(k-1) N} x \in U_{s(k)}$.

For $i \in\{0,1, \ldots, d\}$, denote by $C[i]$ the cylinder set defined by $i$, that is

$$
C[i]=\left\{x \in\{0,1, \ldots, d\}^{\mathbb{N}_{0}}: x_{0}=i\right\} .
$$

Theorem 4.3. Let $(X, f)$ be a non-trivial non-wandering system with the shadowing property. Then the following conditions are equivalents:

(1) $(X, f)$ is weakly mixing;

(2) For every $d \geq 1$ and every non-empty open subsets $U_{0}, U_{1}, \ldots, U_{d}$ of $X$, there exists a positive integer $m \in \mathbb{N}$, a subsystem $\left(Y, f^{m}\right)$ of $\left(X, f^{m}\right)$ and a factor map $\pi:\left(Y, f^{m}\right) \rightarrow\left(\{0,1, \ldots, d\}^{\mathbb{N}_{0}}, \sigma\right)$ such that $\pi^{-1}(C[i]) \subset U_{i}$ for $i=0,1, \ldots, d$;

(3) $(X, f)$ has the strong Property $P$;

(4) $(X, f)$ has uniformly positive entropy of all orders.

Proof. (11) $\Rightarrow$ (2) Choose points $z_{i} \in U_{i}$ for $i=0,1, \ldots, d$ such that $z_{i} \neq z_{j}$ for $i \neq j$. Let $\lambda<\min _{i \neq j} d\left(z_{i}, z_{j}\right)$ with $\bar{B}\left(z_{i}, \lambda\right) \subset U_{i}$ for $i=0,1, \ldots, d$. Let $\varepsilon<\frac{1}{8} \lambda$ and let $\delta>0$ such that every $\delta$-pseudo orbit of $f$ is $\varepsilon$-traced by some point in $X$. Choose a transitive point $\left(u_{0}, u_{1}, \ldots, u_{d}\right)$ in $\left(X^{d+1}, f^{(d+1)}\right)$ with $\max _{i \neq j} d\left(u_{i}, u_{j}\right)<\frac{1}{4} \delta$. Then there exists a positive integer $r$ such that $d\left(f^{r}\left(u_{i}\right), z_{i}\right)<\frac{1}{4} \varepsilon$ for $i=0,1, \ldots, d$, and a positive integer $m>r$ such that $d\left(f^{m}\left(u_{i}\right), u_{i}\right)<\frac{1}{4} \delta$ for $i=0,1, \ldots, d$. 
Define $d+1$ finite sequences as follows

$$
\begin{aligned}
\eta(0) & =\left(u_{0}, f\left(u_{0}\right), \ldots, f^{m-1}\left(u_{0}\right)\right), \\
\eta(1) & =\left(u_{1}, f\left(u_{1}\right), \ldots, f^{m-1}\left(u_{1}\right)\right), \\
& \ldots \\
\eta(d) & =\left(u_{d}, f\left(u_{d}\right), \ldots, f^{m-1}\left(u_{d}\right)\right) .
\end{aligned}
$$

Let $W_{0}=\bar{B}\left(f^{r}\left(u_{0}\right), \varepsilon\right), W_{1}=\bar{B}\left(f^{r}\left(u_{1}\right), \varepsilon\right), \ldots, W_{d}=\bar{B}\left(f^{r}\left(u_{d}\right), \varepsilon\right)$. Then $W_{0}$, $W_{1}, \ldots, W_{d}$ are non-empty closed subsets, and $\operatorname{dist}\left(W_{i}, W_{j}\right)>\varepsilon$ for $i \neq j$. Clearly $W_{i} \subset \bar{B}\left(z_{1}, 2 \varepsilon\right) \subset \bar{B}\left(z_{1}, \lambda\right) \subset U_{i}$.

If $\alpha=a_{0} a_{1} \ldots a_{n} \ldots \in\{0,1, \ldots, d\}^{\mathbb{N}_{0}}$, we set $Y_{\alpha}=\left\{x \in X: f^{m i}(x) \in W_{a_{i}}\right.$ for $i=$ $0,1, \ldots\}$. Then $Y_{\alpha}$ is a closed subset of $X$. We should show that $Y_{\alpha}$ is not empty. Let $y_{\alpha}$ be a point $\varepsilon$-tracing the $\delta$-pseudo orbit $\eta\left(a_{0}\right) \eta\left(a_{1}\right) \cdots \eta\left(a_{n}\right) \cdots$. Then $f^{r}\left(y_{\alpha}\right) \in$ $Y_{\alpha}$. This shows that $Y_{\alpha}$ is not empty. Let $Y=\bigcup_{\alpha \in\{0,1, \ldots, d\}^{\mathbb{N}_{0}}} Y_{\alpha}$ and let $\pi: Y \rightarrow$ $\{0,1, \ldots, d\}^{\mathbb{N}_{0}}, \pi\left(Y_{\alpha}\right)=\alpha$. Then $Y$ is closed and $\pi$ is a factor map between $\left(Y, f^{m}\right)$ and $\left(\{0,1, \ldots, d\}^{\mathbb{N}_{0}}, \sigma\right)$. Finally, note that $\pi^{-1}(C[i])=\bigcup_{\alpha \in\{0,1, \ldots, d\}^{\mathbb{N}_{0}}} Y_{i \alpha} \subset U_{i}$ for $i=$ $0,1, \ldots, d$.

(2) $\Rightarrow$ (3) Fix $n \geq 2$ and non-empty open subsets $U_{0}, U_{1}, \ldots, U_{n-1}$ of $X$. Then there exists a positive integer $m \in \mathbb{N}$ and a subsystem $\left(Y, f^{m}\right)$ and a factor map $\pi:\left(Y, f^{m}\right) \rightarrow$ $\left(\{0,1, \ldots, n-1\}^{\mathbb{N}_{0}}, \sigma\right)$ such that $\pi^{-1}(C[i]) \subset U_{i}$ for $i=0,1, \ldots, n-1$.

For each $k \geq 2$, and $s=(s(1), s(2), \ldots, s(k)) \in\{0,1, \ldots, n-1\}^{k}$, if $z \in\{0,1, \ldots, n-$ $1\}^{\mathbb{N}_{0}}$ has $s$ as its prefix, then for each $x \in \pi^{-1}(z)$ we have $x \in \pi^{-1}(C[s(1)]) \subset$ $U_{s(1)}, f^{m M}(x) \in \pi^{-1}(C[s(2)]) \subset U_{s(2)}, \ldots, f^{(k-1) m M} x \in \pi^{-1}(C[s(k)]) \subset U_{s(k)}$.

(3) $\Rightarrow$ (44) follows from [13, Theorem 7.4].

(41) $\Rightarrow$ (11) follows from [4, Propsition 2].

Definition 4.4. A dynamical system $(X, f)$ is positively expansive (with an expansive constant $\beta>0)$ if for any $x, y \in X$ with $x \neq y$ there is $n>0$ such that $d\left(f^{n}(x), f^{n}(y)\right)>$ $\beta$.

Remark 4.5. If a dynamical system $(X, f)$ is positively expansive with expansive constant $\beta$, then for every pseudo-orbit $\xi$ there is at most one point $(\beta / 2)$-tracing $\xi$. Then it is easy to see that in the proof of Theorem 4.3, every $Y_{\alpha}$ is a singleton, and as a consequence $\pi$ is a conjugacy.

The following fact highlights an important property of (positively) expansive systems (see [3, Theorem 3.4.4.]).

Theorem 4.6 (Topological Decomposition Theorem). Let $(X, f)$ be a positively expansive dynamical system with the shadowing property and assume that $f$ surjective. Then the following properties hold:

(1) $\Omega(f)$ contains a finite sequence of pairwise disjoint $f$-invariant closed subsets $B_{i}(1 \leq i \leq l)$ such that $\Omega(f)=\bigcup_{i=1}^{l} B_{i}$ and each subsystem $\left(B_{i}, f\right)$ is transitive (sets $B_{i}$ are called basic sets).

(2) For every basic set $B$ there is an integer $a>0$ and a finite sequence of pairwise disjoint $f^{a}$-invariant closed sets $C_{i} \subset B(0 \leq i<a)$ such that $B=\bigcup_{i=0}^{a-1} C_{i}$, 
$f\left(C_{i}\right)=C_{i+1}(\bmod a)$ and each subsystem $\left(C_{i}, f^{a}\right)$ is strongly mixing (sets $C_{i}$ are called elementary sets).

Theorem 4.7. Let $(X, f)$ be a non-wandering system with the shadowing property, where $X$ has no isolated points. If $(X, f)$ is positively expansive, then for every $d \geq 1$ and every non-empty open subset $U$ of $X$, there exists a positive integer $m \in \mathbb{N}$ and a subsystem $\left(Y, f^{m}\right)$ with $Y \subset U$ and a conjugacy map $\pi:\left(Y, f^{m}\right) \rightarrow\left(\{0,1, \ldots, d\}^{\mathbb{N}_{0}}, \sigma\right)$.

Proof. Let $U$ be a non-empty open subset of $X$. By the Topological Decomposition Theorem, there is an elementary set $C$ such that $U \cap C \neq \emptyset$. Since $X$ has no isolated points, so does $C$. There exists $a>0$ such that $\left(C, f^{a}\right)$ is strongly mixing. It is easy to see that $\left(C, f^{a}\right)$ is also positively expansive and has the shadowing property. Then the result follows from Theorem 4.3 and Remark 4.5.

Remark 4.8. In Theorem 4.7, we assume that $X$ has no isolated points. If fact, for every non-wandering dynamical system $(X, f)$, we easily obtain that $X$ has at most countably many isolated points and every isolated point in $X$ must be periodic.

\section{SPECIFICATION PROPERTY}

Let $(X, f)$ be a dynamical system. We say that $f$ satisfies the periodic specification property if for any $\varepsilon>0$ there exists $M>0$ such that for any $k \geq 2$, any $k$ points $x_{1}, x_{2}, \ldots, x_{k} \in X$, any non-negative integers $0 \leq a_{1} \leq b_{1}<a_{2} \leq b_{2}<\ldots<a_{k} \leq b_{k}$ with $a_{i}-b_{i-1} \geq M$ for each $i=2,3, \ldots, k$ and any integer $p \geq M+b_{k}-a_{1}$, there exists a periodic point $z \in X$ with $f^{p}(z)=z$ and $d\left(f^{j}(z), f^{j}\left(x_{i}\right)\right)<\varepsilon$ for all $a_{i} \leq j \leq b_{i}$ and $1 \leq i \leq k$. We say that $f$ satisfies the specification property if the point $z$ in the periodic specification property is not requested to be periodic (hence no condition on $p)$.

It was proved first by Bowen (see [7]) that if $(X, f)$ is a weakly mixing system with the shadowing property, then it satisfies the specification property. Moreover, if $(X, f)$ is also positively expansive, then it satisfies the periodic specification property. It is shown in [17] that every non-wandering system with the shadowing property has a dense set of regularly recurrent points. We combine these above results and show that every weak mixing system with the shadowing property also has the following version of specification property. The proof is inspired by Lemma 3.1 in [17].

Theorem 5.1. Let $(X, f)$ be a weakly mixing system with the shadowing property. For any $\varepsilon>0$ there exists $M>0$ such that for any $k \geq 2$, any $k$ points $x_{1}, x_{2}, \ldots, x_{k} \in X$, any non-negative integers $0 \leq a_{1} \leq b_{1}<a_{2} \leq b_{2}<\ldots<a_{k} \leq b_{k}$ with $a_{i}-b_{i-1} \geq M$ for each $i=2,3, \ldots, k$ and any $p \geq M+b_{k}-a_{1}$, there exists $z \in R R(f)$ such that $d\left(f^{j}(z), f^{n p+j}(z)\right)<\varepsilon$ for every $n, j \geq 0$ and $d\left(f^{n p+j}(z), f^{j}\left(x_{i}\right)\right)<\varepsilon$ for all $a_{i} \leq j \leq b_{i}$, $1 \leq i \leq k$ and $n \geq 0$.

Proof. First note that we may assume that $a_{1}=0$. Simply, if $z^{\prime}$ is a point obtained for $a_{i}^{\prime}=a_{i}-a_{1}, b_{i}^{\prime}=b_{i}-a_{1}$ and $x_{i}^{\prime}=f^{a_{1}}\left(x_{i}\right)$ then $z=f^{p-a_{1}}\left(z^{\prime}\right)$ is a desired point.

Let $\lambda_{0}=\varepsilon / 8$ and let $\delta>0$ be such that every $\delta$-pseudo-orbit is $\lambda_{0} / 8$-traced. Since $f$ is weakly mixing, by results of [19] it is chain mixing, that is, there is $M>0$ such that for any two points $x, y \in X$ and any $n \geq M$ there is a $\delta$-chain of length $n$ from $x$ to $y$. 
Now fix $k, p$ and sequences $x_{i}, a_{i}, b_{i}$ as in the statement of theorem (with $M$ fixed above). There is a periodic $\delta$-pseudo-orbit

$$
\xi=\left(z_{0}, \ldots, z_{p-1}, z_{0}, \ldots z_{p-1}, z_{0}, \ldots\right)
$$

such that $z_{j}=f^{j}\left(x_{i}\right)$ for $i=1, \ldots, k$ and all $a_{i} \leq j \leq b_{i}$. Let $q_{0}$ be a point which is $\lambda_{0} / 8$-tracing $\xi$. Let $y_{0}$ be any minimal point in the set $\omega\left(q_{0}, f^{p}\right)$. For any $i \geq 0$ there is $\tau>0$ such that $d\left(f^{i}\left(y_{0}\right), f^{\tau p+i}\left(q_{0}\right)\right)<\lambda_{0} / 8$. Then

$$
\begin{aligned}
d\left(f^{i}\left(y_{0}\right), \xi_{i}\right) & \leq d\left(f^{i}\left(y_{0}\right), f^{\tau p+i}\left(q_{0}\right)\right)+d\left(\xi_{i}, f^{\tau p+i}\left(q_{0}\right)\right)<\lambda_{0} / 8+\lambda_{0} / 8 \\
& \leq \lambda_{0} / 4
\end{aligned}
$$

which implies that $y_{0}$ is $\lambda_{0} / 4$-tracing $\xi$.

Denote $\lambda_{r}=\varepsilon / 8^{r+1}$ for $r=0,1,2, \ldots$ and $m_{0}=p$. Now we will apply arguments inspired by the proof of Lemma 3.1 in [17, and construct an increasing sequence $\left\{m_{r}\right\}_{r=0}^{\infty}$ of natural numbers and a sequence $\left\{y_{r}\right\}_{r=0}^{\infty}$ of points of $X$ such that for every $r=0,1,2, \ldots$ we have

(1) $m_{r}$ divides $m_{r+1}$;

(2) $y_{r}$ is a minimal point and $d\left(f^{i m_{r}+j}\left(y_{r}\right), f^{j}\left(y_{r}\right)\right)<\frac{\lambda_{r+1}}{2}$ for any $i, j \geq 0$;

(3) $d\left(f^{j}\left(y_{r}\right), f^{j}\left(y_{l-1}\right)\right) \leq \sum_{i=l}^{r} \lambda_{i}$ for $1 \leq l \leq r$ and every $j \geq 0$.

Before we proceed with the construction, let us first assume that we have already constructed a sequence of points $\left\{y_{r}\right\}$ satisfying (11)-(3). Let $z$ be a limit point of the sequence $\left\{y_{r}\right\}$. For every $j \geq 0$ there is $s>0$ such that $d\left(f^{j}(z), f^{j}\left(y_{i_{s}}\right)\right)<\varepsilon / 8$ and then (applying condition (3) $)$ we obtain that

$$
\begin{aligned}
d\left(f^{j}(z), \xi_{j}\right) & \leq d\left(\xi_{j}, f^{j}\left(y_{0}\right)\right)+d\left(f^{j}\left(y_{0}\right), f^{j}\left(y_{i_{s}}\right)\right)+d\left(f^{j}\left(y_{i_{s}}\right), f^{j}(z)\right) \\
& \leq \frac{\varepsilon}{8}+\sum_{i=1}^{i_{s}} \lambda_{i}+\frac{\varepsilon}{8} \leq \frac{\varepsilon}{4}+\sum_{i=1}^{\infty} \lambda_{i}<\frac{\varepsilon}{2}
\end{aligned}
$$

In particular, for any $n \geq 0$ we have

$$
\begin{aligned}
d\left(f^{j}(z), f^{n p+j}(z)\right) & \leq d\left(f^{j}(z), \xi_{j}\right)+d\left(\xi_{j}, f^{n p+j}(z)\right) \\
& =d\left(f^{j}(z), \xi_{j}\right)+d\left(\xi_{n p+j}, f^{n p+j}(z)\right) \\
& <\varepsilon
\end{aligned}
$$

Similarly, for every $r \geq 1$ and every $j \geq 0$ there is $s>r$ such that $d\left(f^{j m_{r}}(z), f^{j m_{r}}\left(y_{i_{s}}\right)\right)<$ $\lambda_{r}$ and $d\left(z, y_{i_{s}}\right)<\lambda_{r}$. Clearly $i_{s} \geq s$. Additionally, observe that (3) implies

$$
\begin{aligned}
\left.d\left(y_{i_{s}}, y_{r}\right)\right) & \leq \sum_{i=r+1}^{i_{s}} \lambda_{i}, \\
\left.d\left(f^{j m_{r}}\left(y_{i_{s}}\right), f^{j m_{r}}\left(y_{r}\right)\right)\right) & \leq \sum_{i=r+1}^{i_{s}} \lambda_{i}
\end{aligned}
$$


and by (2) we also have that $d\left(f^{j m_{r}}\left(y_{r}\right), y_{r}\right)<\frac{\lambda_{r+1}}{2}$ which gives the following

$$
\begin{aligned}
d\left(f^{j m_{r}}(z), z\right) \leq & d\left(f^{j m_{r}}(z), f^{j m_{r}}\left(y_{i_{s}}\right)\right)+d\left(f^{j m_{r}}\left(y_{i_{s}}\right), f^{j m_{r}}\left(y_{r}\right)\right) \\
& +d\left(f^{j m_{r}}\left(y_{r}\right), y_{r}\right)+d\left(y_{r}, y_{i_{s}}\right)+d\left(y_{i_{s}}, z\right) \\
\leq & \lambda_{r}+2 \sum_{i=r+1}^{i_{s}} \lambda_{i}+\frac{\lambda_{r+1}}{2}+\lambda_{r} \leq 3 \sum_{i=r}^{\infty} \lambda_{i} .
\end{aligned}
$$

But $\lim _{r \rightarrow \infty} \sum_{i=r}^{\infty} \lambda_{i}=0$, hence $z \in R R(f)$.

To complete the proof, we need to perform a construction of the sequences $\left\{m_{r}\right\}$ and $\left\{y_{r}\right\}$ satisfying (1)-(3). Fix $s \geq 0$ and suppose that $m_{r}, y_{r}$ are constructed for every $0 \leq r \leq s$. We will show, how $m_{s+1}$ and $y_{s+1}$ can be constructed. Let $\alpha>0$ be such that every $\alpha$-pseudo-orbit is $\lambda_{s+2} / 8$-traced. Since $y_{s}$ is minimal for $f$, it is also minimal for $f^{m_{s}}$, in particular, there is a positive integer $m_{s+1}$ divisible by $m_{s}$ such that $d\left(f^{m_{s+1}}\left(y_{s}\right), y_{s}\right)<\alpha$. Take a periodic $\alpha$-pseudo-orbit

$$
\gamma=\left(y_{s}, f\left(y_{s}\right), \ldots, f^{m_{s+1}-1}\left(y_{s}\right), y_{s}, f\left(y_{s}\right), \ldots, f^{m_{s+1}-1}\left(y_{s}\right), y_{s}, \ldots\right)
$$

and let $q_{s+1}$ be a point which is $\lambda_{s+2} / 8$-tracing $\gamma$. Let $y_{s+1}$ be any minimal point in the set $\omega\left(q_{s+1}, f^{m_{s+1}}\right)$. If we fix any $i, j \geq 0$ then there is $\tau>0$ such that $d\left(f^{i m_{s+1}+j}\left(y_{s+1}\right), f^{(i+\tau) m_{s+1}+j}\left(q_{s+1}\right)\right)<\lambda_{s+2} / 8$ and $d\left(f^{j}\left(y_{s+1}\right), f^{\tau m_{s+1}+j}\left(q_{s+1}\right)\right)<\lambda_{s+2} / 8$. But then

$$
\begin{aligned}
d\left(f^{i m_{s+1}+j}\left(y_{s+1}\right), f^{j}\left(y_{s+1}\right)\right) & <d\left(f^{i m_{s+1}+j}\left(y_{s+1}\right), \gamma_{\tau m_{s+1}+j}\right)+d\left(\gamma_{\tau m_{s+1}+j}, f^{j}\left(y_{s+1}\right)\right) \\
& =d\left(f^{i m_{s+1}+j}\left(y_{s+1}\right), \gamma_{(i+\tau) m_{s+1}+j}\right)+d\left(\gamma_{\tau m_{s+1}+j}, f^{j}\left(y_{s+1}\right)\right) \\
& <\frac{\lambda_{s+2}}{8}+\frac{\lambda_{s+2}}{8}+\frac{\lambda_{s+2}}{8}+\frac{\lambda_{s+2}}{8}=\frac{\lambda_{s+2}}{2}
\end{aligned}
$$

which proves (2). Similarly, fix any $1 \leq l \leq s+1$ and any $j \geq 0$, there is $\tau>0$ such that $d\left(f^{j}\left(y_{s+1}\right), f^{\tau m_{s+1}+j}\left(q_{s+1}\right)\right)<\lambda_{s+2} / 8$. There is $0 \leq t<m_{s+1}$ and $i \geq 0$ such that $j=i m_{s+1}+t$ and then

$$
\begin{aligned}
d\left(f^{j}\left(y_{s+1}\right), f^{j}\left(y_{l-1}\right)\right) & \leq d\left(f^{j}\left(y_{s+1}\right), \gamma_{\tau m_{s+1}+j}\right)+d\left(\gamma_{\tau m_{s+1}+j}, f^{j}\left(y_{l-1}\right)\right) \\
& \leq d\left(f^{j}\left(y_{s+1}\right), \gamma_{\tau m_{s+1}+j}\right)+d\left(\gamma_{t}, f^{j}\left(y_{s}\right)\right)+d\left(f^{j}\left(y_{s}\right), f^{j}\left(y_{l-1}\right)\right) \\
& \leq \frac{\lambda_{s+2}}{8}+\frac{\lambda_{s+2}}{8}+d\left(f^{t}\left(y_{s}\right), f^{i m_{s+1}+t}\left(y_{s}\right)\right)+\sum_{i=l}^{s} \lambda_{i} \\
& \leq \frac{\lambda_{s+2}}{4}+\frac{\lambda_{s+1}}{2}+\sum_{i=l}^{s} \lambda_{i}<\sum_{i=l}^{s+1} \lambda_{i},
\end{aligned}
$$

which proves (3) and ends the proof.

As an easy consequence of Theorem 5.1 we obtain a classical result, proved first by Bowen (see [7]).

Corollary 5.2. If $(X, f)$ is weakly mixing, positively expansive and has the shadowing property, then it has the periodic specification property. 
Proof. Fix $\varepsilon<\beta$ where $\beta$ is an expansive constant. The only thing which we need to extend in Theorem 5.1 to obtain periodic specification property is that $z$ is a periodic point with period $p$. But if $\varepsilon<\beta$ then in Theorem 5.1 for every $j \geq 0$ we have

$$
d\left(f^{j}(z), f^{j+p}(z)\right) \leq \varepsilon<\beta .
$$

Hence $z=f^{p}(z)$ completing the proof.

Given a compact metric space $X$, denote by $C(X, \mathbb{R})$ the set of all continuous functions $\xi: X \rightarrow \mathbb{R}$. If we endow it with the norm $\|\xi\|=\sup _{x \in X}|\xi(x)|$ then it becomes a Banach space.

Let $\mathcal{M}(X)$ be the set of all Borel probability measures on $X$ and fix a dense sequence $\left\{\xi_{i}\right\}_{i=1}^{\infty} \subset C(X, \mathbb{R})$. Then the metric

$$
\mathcal{D}(\mu, \nu)=\sum_{i=1}^{\infty} \frac{\left|\int_{X} \xi_{i} d \mu-\int_{X} \xi_{i} d \nu\right|}{2^{i}\left(\left\|\xi_{i}\right\|+1\right)}
$$

for $\mu, \nu \in \mathcal{M}(X)$ is compatible with the weak-* topology on $\mathcal{M}(X)$. We denote by $\mathcal{M}_{f}(X) \subset \mathcal{M}(X)$ the set of all invariant measures for $(X, f)$. The support of a measure $\mu \in M(X)$, denoted by $\operatorname{supp}(\mu)$, is the smallest closed subset $C$ of $X$ such that $\mu(C)=1$. If $\mu$ is an invariant measure for $(X, f)$, then it is clear that $\operatorname{supp}(\mu)$ is $f$-invariant.

Let $x \in X$ be a periodic point with periodic $p$. Then it corresponds to an invariant measure $\mu_{x}$ which has mass $1 / p$ as each of the points $x, f(x), \ldots, f^{p-1}(x)$. We denote the set of these measures by $P(p)$. The following fact was first proved by Sigmund (see [20]).

Theorem 5.3. If $(X, f)$ satisfies the periodic specification property and if $\ell \in \mathbb{N}$, then $\bigcup_{p \geq \ell} P(p)$ is dense in $\mathcal{M}_{f}(X)$.

While there are known examples of weakly mixing systems with the shadowing property that have no periodic points (e.g. see Example 5.6), we can prove that the assertion of Theorem 5.3 can be preserved to some extent. Strictly speaking, we can show that if a weakly mixing system has the shadowing property, then the set of ergodic invariant measures supported on the closures of orbits of regularly recurrent points is dense in the set of invariant measures.

Definition 5.4. Denote by $E_{f}^{T}(X)$ the set of ergodic measures $\mu \in \mathcal{M}_{f}(X)$ for $(X, f)$ such that the support of $\mu$ is the closure of the orbit of a regularly recurrent point.

Denote by $Q(f)$ the set of quasi-regular points with respect to $f$, that is the set of points $x$ such that the limit

$$
\xi^{*}(x)=\lim _{n \rightarrow \infty} \frac{1}{n} \sum_{j=0}^{n-1} \xi\left(f^{j}(x)\right)
$$

exists for every $\xi \in C(X, \mathbb{R})$. It can be proved that $Q(f)$ is a Borel set and that $\mu(Q(f))=1$ for every $\mu \in \mathcal{M}_{f}(X)$ (e.g. see [3]).

The proof of the following fact is in main part the same as original Sigmund's argument in the proof of Theorem 5.3. The final argument is made by application of 
Theorem 5.1. Since it could be hard to present rigorous explanation of the proof without detailed reference to [20], we decided to provide a complete proof. It makes the paper complete (and hence accessible to the reader), and at the same time fits into approach presented by various authors before, when proving variants of Sigmund's result (e.g. see [9]).

Corollary 5.5. Let $(X, f)$ be a weakly mixing system with the shadowing property. Then $E_{f}^{T}(X)$ is a dense subset of $\mathcal{M}_{f}(X)$.

Proof. Fix any $\mu \in \mathcal{M}_{f}(X)$ and its open neighborhood $U$. By the definition of metric $\mathcal{D}$ there is $\varepsilon>0$ and a finite set $F \subset C(X, \mathbb{R})$, such that $\|\xi\| \leq 1$ for all $\xi \in F$ and

$$
W=\left\{\nu \in \mathcal{M}_{f}(X):\left|\int_{X} \xi d \mu-\int_{X} \xi d \nu\right|<\varepsilon \text { for all } \xi \in F\right\} \subset U .
$$

By Birkhoff Ergodic Theorem and the fact that $\mu(Q(f))=1$ we have for each $\xi \in F$

$$
\int_{Q(f)} \xi d \mu=\int_{Q(f)} \xi^{*} d \mu
$$

It is clear that $\left.\xi^{*}\right|_{Q(f)}$ is Borel and $\sup _{x \in Q(f)}\left|\xi^{*}(x)\right| \leq 1$ for all $\xi \in F$. Let $\mathcal{P}=$ $\left\{P_{1}, \ldots, P_{s}\right\}$ be a partition of $Q(f)$ into non-empty Borel sets such that $\left.\xi^{*}\right|_{P_{i}}$ has oscillation bounded by $\varepsilon / 4$ for all $\xi \in F$ and $i=1, \ldots, s$ (i.e. $\sup _{x \in P_{i}} \xi^{*}(x)-\inf _{y \in P_{i}} \xi^{*}(y) \leq$ $\varepsilon / 4)$.

For each $j=1, \ldots, s$, choose a point $y_{j} \in P_{s}$ and observe that

$$
\left|\int_{P_{j}} \xi^{*} d \mu-\mu\left(P_{j}\right) \xi^{*}\left(y_{j}\right)\right| \leq \frac{\varepsilon}{4} \mu\left(P_{j}\right)
$$

which immediately implies that

$$
\left|\int_{Q(f)} \xi d \mu-\sum_{j=1}^{s} \mu\left(P_{j}\right) \xi^{*}\left(y_{j}\right)\right| \leq \frac{\varepsilon}{4} .
$$

There is $\delta>0$ such that if $d\left(z_{1}, z_{2}\right)<\delta$ then $\left|\xi\left(z_{1}\right)-\xi\left(z_{2}\right)\right|<\varepsilon / 8$, provided that $\xi \in F$ and $z_{1}, z_{2} \in X$. Let $M$ be provided by Theorem 5.1 for $\delta / 4$.

Directly from the definition of $Q(f)$ we can find $N>0$ such that for any $\xi \in F$ and $j=1, \ldots, s$ we have

In particular we have

$$
\left|\frac{1}{N} \sum_{i=0}^{N-1} \xi\left(f^{i}\left(y_{j}\right)\right)-\xi^{*}\left(y_{j}\right)\right|<\frac{\varepsilon}{8} .
$$

$$
\left|\int_{Q(f)} \xi d \mu-\frac{1}{N} \sum_{j=1}^{s} \sum_{i=0}^{N-1} \mu\left(P_{j}\right) \xi\left(f^{i}\left(y_{j}\right)\right)\right| \leq \frac{3 \varepsilon}{8}
$$

Fix a positive integer $m$ such that $\frac{1}{m}<\frac{\varepsilon}{8 s}$. There exist positive integers $n_{1}, \ldots, n_{s}$ such that for $j=1, \ldots, s$ we have

$$
\frac{n_{j}}{m} \leq \mu\left(P_{j}\right)<\frac{n_{j}+1}{m}
$$


Putting $m_{j}=n_{j}$ or $m_{j}=n_{j}+1$ we can present $\sum_{j=1}^{s} m_{j}=m$ and clearly we also have

$$
\left|\mu\left(P_{j}\right)-\frac{m_{j}}{m}\right| \leq \frac{1}{m} \leq \frac{\varepsilon}{8 s} .
$$

Therefore

$$
\left|\frac{1}{N} \sum_{j=1}^{s} \sum_{i=0}^{N-1}\left(\mu\left(P_{j}\right)-\frac{m_{j}}{m}\right) \xi\left(f^{i}\left(y_{j}\right)\right)\right| \leq \frac{\varepsilon}{8 N} \sum_{i=0}^{N-1}\left|\xi\left(f^{i}\left(y_{j}\right)\right)\right| \leq \frac{\varepsilon}{8}
$$

which combined with (5.1) implies that

$$
\left|\int_{Q(f)} \xi d \mu-\frac{1}{N} \sum_{j=1}^{s} \sum_{i=0}^{N-1} \frac{m_{j}}{m} \xi\left(f^{i}\left(y_{j}\right)\right)\right| \leq \frac{\varepsilon}{2} .
$$

Increasing $N$ if necessary, we may assume that $2 M / N<\varepsilon / 8$.

Since $f$ is weakly mixing, it is onto and hence for every $a>0$ and $j$ there is $x_{j}$ such that $f^{a}\left(x_{j}\right)=y_{j}$. For $r=1, \ldots, m$ we put $a_{r}=(r-1)(N+M), b_{r}=a_{r}+N-1$ and let $x_{r}$ be such that $f^{a_{r}}\left(x_{r}\right)=y_{j}$ where $1 \leq j \leq s$ is the largest number such that $\sum_{i=1}^{j-1} m_{j} \leq r-1$. Put $p=m(N+M)$. Then

$$
\left|\frac{1}{p} \sum_{r=1}^{m} \sum_{i=a_{r}}^{b_{r}} \xi\left(f^{i}\left(x_{r}\right)\right)-\frac{1}{N} \sum_{j=1}^{s} \sum_{i=0}^{N-1} \frac{m_{j}}{m} \xi\left(f^{i}\left(y_{j}\right)\right)\right| \leq \frac{M}{N+M}<\frac{\varepsilon}{16} .
$$

Let $z$ be provided by Theorem 5.1 for $\delta / 4$, the sequences $a_{r}, b_{r}$, points $x_{r}$ and $p$. Put $\Lambda=\omega(z, f)$ and take an ergodic measure $\nu$ on $\Lambda$. Since $\nu$ is ergodic, there exists a point $q \in \Lambda$ such that

$$
\lim _{n \rightarrow \infty} \frac{1}{n} \sum_{i=0}^{n-1} \xi\left(f^{i}(q)\right)=\int_{\Lambda} \xi d \nu=\int_{Q(f)} \xi d \nu
$$

for each $\xi \in F$. Taking forward iteration of $q$ if necessary, we may assume that $q \in$ $\omega\left(z, f^{p}\right)$. For every $j \geq 0$, there exists $\tau>0$ such that $d\left(f^{j} p, f^{\tau p+j}(z)\right)<\delta / 4$, and then

$$
\begin{aligned}
d\left(f^{j}(q), f^{j}(z)\right) & \leq d\left(f^{j}(q), f^{\tau p+j}(z)\right)+d\left(f^{\tau p+j}(z), f^{j}(z)\right) \\
& <\delta / 4+\delta / 4=\delta / 2 .
\end{aligned}
$$

Therefore $d\left(f^{i}(q), f^{i}\left(x_{r}\right)\right)<\delta$ for all $a_{r} \leq i \leq b_{r}$ and $1 \leq r \leq m$,

Choose an integer $T>0$ such that for each $\xi \in F$ we have

$$
\left|\frac{1}{p T} \sum_{i=0}^{p T-1} \xi\left(f^{i}(q)\right)-\int_{Q(f)} \xi d \nu\right|<\frac{\varepsilon}{8} .
$$

By the choice of $p$, we have $d\left(f^{n p+i}(q), f^{i}(q)\right) \leq \delta$ for all $i, n \geq 0$, and hence

$$
\left|\frac{1}{p} \sum_{i=0}^{p-1} \xi\left(f^{i}(q)\right)-\int_{Q(f)} \xi d \nu\right| \leq \frac{\varepsilon}{8}+\left|\frac{1}{p} \sum_{i=0}^{p-1} \xi\left(f^{i}(q)\right)-\frac{1}{p T} \sum_{i=0}^{p T-1} \xi\left(f^{i}(q)\right)\right|<\frac{\varepsilon}{4} .
$$


By our construction we have

$$
\left|\frac{1}{p} \sum_{r=1}^{m} \sum_{i=a_{r}}^{b_{r}}\left(\xi\left(f^{i}(q)\right)-\xi\left(f^{i}\left(x_{r}\right)\right)\right)\right| \leq \frac{m N \varepsilon}{8 p} \leq \frac{\varepsilon}{8},
$$

and finally

$$
\left|\frac{1}{p} \sum_{r=1}^{m} \sum_{i=b_{r}+1}^{b_{r}+M} \xi\left(f^{i}(q)\right)\right| \leq \frac{M m}{p} \leq \frac{\varepsilon}{16} .
$$

Now, combining the above calculations (5.2)-(5.6), we obtain that

$$
\left|\int_{X} \xi d \mu-\int_{X} \xi d \nu\right|<\frac{\varepsilon}{2}+\frac{\varepsilon}{16}+\frac{\varepsilon}{8}+\frac{\varepsilon}{16}+\frac{\varepsilon}{4} \leq \varepsilon .
$$

This shows that $\nu \in W$ completing the proof.

We finish our considerations with a few simple examples. Note that if a dynamical system is positively expansive, then Theorem 5.5 is a consequence of Theorem 5.3 by Corollary [5.2. The same is also true for other types of expansiveness, e.g. expansive homeomorphism or $c$-expansive surjections as defined in [3]. We leave details to the reader. A large class of systems with the shadowing property which are not positively expansive can be found within the class of dynamical systems on the unit interval, e.g. in the family of tent maps (e.g. see [6]) In these maps, however, another argument can be used to prove the periodic specification property (see Buzzi's proof of Blokh's classical result in [5]), so ergodic measures in Theorem 5.5 are in fact supported on these points, since Theorem 5.3 works.

To obtain a system which satisfies assumption of Theorem 5.5 but not of Theorem 5.3 we need a little more work. To do so, we can perform the following standard construction.

Example 5.6. Let $\left(X_{n}, f_{n}\right)$ be a strongly mixing system with the shadowing property but without points of period n. For example it can be a subshift of finite type defined by a graph with two distinct cycles of length $n+1$ and $n+2$ starting from the same vertex. Assume that the metric on $\left(X_{n}, d_{n}\right)$ satisfies $\operatorname{diam}\left(X_{n}\right) \leq 1$. If we take an infinite Cartesian product $(X, F)=\left(\prod_{n=1}^{\infty} X_{n}, \prod_{n=1}^{\infty} f_{n}\right)$ with the standard product metric $d(x, y)=\sum_{n=1}^{\infty} 2^{-n} d_{n}\left(x_{n}, y_{n}\right)$ then it generates a topology compatible with the Thikhonov topology, in particular it is compact. The map $(X, F)$ is strongly mixing and has the shadowing property as a product of maps with the same properties. But it cannot have periodic points, because for any $x \in X$ and $n \in \mathbb{N}$ we have $F^{n}(x)_{n}=f^{n}\left(x_{n}\right) \neq x_{n}$.

In the above example the set consisting of regularly recurrent points whose orbits closures form subsystems conjugate to odometers is dense.

Question 1. Can we ensure in Theorem 5.1 that $z \in R R(f)$ is such that $\overline{\operatorname{Orb}(z, f)}$ is an odometer (up to conjugacy), not only its almost 1-1 extension?

Note that if we can prove in Theorem 5.1 that $z$ is not only regularly recurrent but also equicontinuous (in its orbit closure), then immediately Question 1 has a positive answer. 
Acknowledgement. The first author was partially supported by STU Scientific Research Foundation for Talents (NTF12021), Guangdong Natural Science Foundation (S2013040014084) and NNSF of China (11171320). The research of second author was supported by Narodowe Centrum Nauki (National Science Center) in Poland, grant no. DEC-2011/03/B/ST1/00790.

The authors would like to thank Guohua Zhang for his helpful remarks. The authors express many thanks to the anonymous referee, whose remarks resulted in substantial improvements of the paper.

\section{REFERENCES}

[1] E. Akin, On chain continuity, Discrete Contin. Dyn. Syst., 2 (1996), 111-120.

[2] E. Akin, J. Auslander, K. Berg, When is a transitive map chaotic? in: Convergence in Ergodic Theory and Probability, Columbus, OH, 1993, in: Ohio State Univ. Math. Res. Inst. Publ., vol. 5, de Gruyter, Berlin, 1996, pp. 25-40.

[3] N. Aoki and K. Hiraide, Topological Theory of Dynamical Systems, North-Holland, 1994.

[4] F. Blanchard, Fully positive topological entropy and topological mixing. Symbolic dynamics and its applications (New Haven, CT, 1991), 95-105, Contemp. Math., 135, Amer. Math. Soc., Providence, RI, 1992.

[5] J. Buzzi, Specificaiton on the interval, Trans. Amer. Math. Soc, 349, (1997), 2737-2754

[6] E. M. Coven, I. Kan and J. A. Yorke, Pseudo-orbit shadowing in the family of tent maps, Trans. Amer. Math. Soc., 308(1) (1988), 227-241.

[7] M. Denker, C. Grillenberger and K. Sigmund, Ergodic theory on compact spaces. Lecture Notes in Mathematics, Vol. 527. Springer-Verlag, Berlin-New York, 1976.

[8] T. Downarowicz, Survey of odometers and Toeplitz flows, algebraic and topological dynamics, vol. 385, pp. 7-37. Contemporary Mathematics-American Mathematics Society, Providence (2005).

[9] F. Hofbauer, Generic properties of invariant measures for simple piecewise monotonic transformations, Israle J. Math, 59 (1987), 64-80.

[10] E. Glasner and B. Weiss, Sensitive dependence on initial conditions, Nonlinearity 6 (1993), 10671075.

[11] E. Glasner and B. Weiss, Kazhdan's property $T$ and the geometry of the collection of invariant measures, Geometry and Functional Analysis, 7 (1997), 917-935.

[12] L. Chen and S.H. Li, Shadowing property for inverse limit spaces, Proc. Amer. Math. Soc., 115 (1992), 573-580.

[13] W. Huang, X. Ye, A local variational relation and applications. Israel J. Math. 151 (2006), 237279.

[14] J. Li, Chaos and entropy for interval maps, J. Dyn. Diff. Equat., 23 (2011), no. 2, 333-352.

[15] J. Lindenstrauss, G. Olsen and Y. Sternfeld, The Poulsen Simplex, Ann. Inst. Fourier, Grenoble, 28, 1 (1978), 91-114.

[16] T.K.S. Moothathu, Implications of pseudo-orbit tracing property for continuous maps on compacta, Top. Appl., 158 (2011), 2232-2239.

[17] T.K.S. Moothathu and P. Oprocha, Shadowing entropy and minimal subsystems, Monatsh. Math, in press, DOI: $10.1007 / \mathrm{s} 00605-013-0504-3$

[18] J. Mai,and X. Ye, The structure of pointwise recurrent maps having the pseudo orbit tracing property, Nagoya Math. J. 166, 83-92 (2002)

[19] D. Richeson and J. Wiseman, Chain recurrence rates and topological entropy, Topology Appl. 156 (2008), 251-261.

[20] K. Sigmund, Generic properties of invariant measures for axiom-A diffeomorphisms, Inventiones math. 11 (1970), 99-109.

[21] P. Walters, An introduction to ergodic theory, Graduate Texts in Mathematics, vol. 79, SpringerVerlag, New York, 1982. 
[22] X. Ye, G. Zhang, Entropy points and applications, Trans. Amer. Math. Soc. 359 (12) (2007), 6167-6186.

(J. Li) Department of Mathematics, Shantou University, Shantou, Guangdong, 515063, P.R. CHINA

E-mail address: lijian09@mail.ustc.edu.cn

(P. Oprocha) AGH University of Science and Technology, Faculty of Applied Mathematics, al. Mickiewicza 30, 30-059 Kraków, Poland - And - Institute of Mathematics, Polish Academy of Sciences, ul. Śniadeckich 8, 00-956 Warszawa, Poland

E-mail address: oprocha@agh.edu.pl 